\title{
Childbirth experience and practice changing during COVID-19 pandemic: a prospective cohort study.
}

\author{
Annalisa Inversetti ${ }^{1}$, Simona Fumagalli ${ }^{2}$, Antonella Nespoli $^{2}$, Laura Antolini ${ }^{2}$, Serena \\ Mussi $^{1}$, Debora Ferrari ${ }^{2}$, and Anna Locatelli ${ }^{2}$ \\ ${ }^{1}$ Carate Brianza Hospital, ASST Vimercate \\ ${ }^{2}$ University of Milan-Bicocca
}

August 14, 2020

\begin{abstract}
Objective: To compare mothers' satisfaction at birth and intrapartum care during COVID-19 pandemic compared to a preCOVID cohort. Design: Prospective cohort study Setting: Low risk maternity unit in Northern Italy Population: Women who delivered during COVID-19 pandemic compared to a pre-COVID cohort recruited in the same setting in February-May, 2018. Methods: Italian version of the Birth Satisfaction Scale-Revised (I-BSS-R) was used. Main Outcome Measures: Satisfaction at birth in relation to socio-demographic characteristics, obstetric history and intrapartum care variables. Results: 377 women were included (277 pre-COVID and 100 during COVID pandemic). A higher rate of induction [40\%, COVID versus 25\% pre-COVID, p 0.004], fewer active phases $>12 \mathrm{~h}$ [6\% COVID versus $15 \%$ pre-COVID, p 0.018] and more acceleration with oxytocin [35\% COVID versus $24.9 \%$ pre-COVID, p 0.05] were found. No differences in terms of satisfaction at birth were reported (I-BSS-R mean 27.0, SD 5.3 versus mean 27.6, SD 6.1, p 0.34). Intrapartum variables that significantly reduced maternal satisfaction were the same in the two groups: epidural analgesia ( $<<0.0001$ in both groups), prolonged active phases ( $<0.0001$ in both), oxytocin administration $(\mathrm{p}<0.0001$ in both) and operative delivery ( 0.0009 versus $\mathrm{p} 0.0019$ ). The lowest scores in COVID era were found in those who underwent induction of labor, active phases $>12 \mathrm{~h}$ and CS in labor. Conclusions: No differences were reported concerning global satisfaction at birth, despite an increased rate of active intrapartum intervention. Keywords: Childbirth experience, COVID-19, intrapartum intervention
\end{abstract}

\section{Funding}

No external funding was used in the conduct of this study.

Tweetable abstract: No differences were reported in terms of satisfaction at birth in COVID compared to pre-COVID era in the same context.

\section{Introduction}

Women's satisfaction with their childbirth experience has short and long-term consequences for women and babies' health, in terms of a future good relationship with the newborn. This process can also influence the attitude towards motherhood that contributes to the woman's sense of competence and confidence ${ }^{1-3}$.

The recent epidemic from a new coronavirus isolated in Wuhan (China) at the end of 2019 (SARS-CoV2 ), among other clinical and public health problems, also raised those related to the perception of labor and birth, in relation to the preventative measures in pregnancy/labor/birth, the possible maternal-infant transmission of the infection, the safety of the management of the mother-neonate and breastfeeding. Within Europe, Italy, and in particular the Northern region Lombardy, was the most affected place ${ }^{4-5}$.

In 2018, two years before the spreading of the infection, we performed a cross-sectional study involving 277 women who delivered in a low risk maternity unit in Northern Italy ${ }^{6}$. Satisfaction with birth was measured 
using the Italian version of the Birth Satisfaction Scale-Revised (I-BSS-R). We showed that intrapartum interventions were negatively associated with maternal outcomes and, therefore, also with maternal satisfaction at birth. Variables significantly associated with the I-BSS-R total score affected the three Sub-Scales (Quality of Care [QC], Women personal Attribute [WA] and Stress Experienced [SE]) differently. For example, multi- parity, antenatal class attendance and intact perineum variables were significantly associated only with the SE Sub-Scale and were not involved with the other two Sub-Scales. Epidural analgesia, oxytocin administration and the active phase of labour being greater than $12 \mathrm{~h}$ were significantly associated with both the SE and WA Sub-Scales and not with the QC Sub-Scale.

The aim of this study is to compare mothers' satisfaction at birth and intrapartum care in a cohort of women who delivered during COVID-19 pandemic to a pre-COVID cohort of 2018 in the same setting and in relation to socio-demographic characteristics, obstetric history and obstetric care variables.

\section{Material and Methods}

\section{Design}

We performed a prospective cohort study in a low risk Maternity Unit in Northern Italy.

2. Setting and adopted measures

The study was conducted in a Level I Maternity Hospital in Northern Italy. The research site has approximately 1300 births per year and one of the lowest rates of caesarean section in Italy. The overall caesarean section (CS) rate is $15.9 \%$ (compared with the national CS rate of $35.4 \%$ ), of which $5 \%$ are primary CS that are performed on women giving birth for the first time.

In the hospital's labour ward there are four rooms, where women remain for two hours following birth, before being transferred to the postnatal ward, where there are twenty-six beds. During the study period, women were discharged 48 hours (h) following a vaginal birth and within $72 \mathrm{~h}$ if a caesarean section occurred compared to the usual $72 \mathrm{~h}$ for both categories. Midwifery care was provided in both labour and postnatal wards.

There were 23 midwives working on the labour ward, 3 midwives per shift with a mean number of 4 births per day (planned CS are included in this number).

The research site was committed to offer a one-midwife-to-one-woman ratio for all women in labour.

COVID-19 pandemic raised the need for a deep reorganization in order to guarantee the safety of mothers, neonates, and healthcare providers.

In particular this consisted in the adoption of the following measures:

- Obstetric emergency of the maternity department provided a triage area ensuring a place of isolation (room with bathroom) and trained staff, equipped with personal protective equipment (PPE).

- Checklist for symptoms was mandatory both for mothers and birth partners, in order to assess symptoms in the 14 days before (fever, acute persistent cough, hoarseness, nasal discharge/ congestion, shortness of breath, sore throat, wheezing or sneezing) and highly risk contacts.

- Distancing measures (at least 1 meter) between patients were guaranteed.

- During the admission to the delivery rooms, mothers and birth partners had to wear surgical masks and gloves. Surgical masks had not to be removed, even during labour and birth.

- Nasopharyngeal swab for COVID-19 was performed to every woman at the admission for delivery or planned $48 \mathrm{~h}$ before (if the admission was planned).

- Before the confirmation of the laboratory, women were managed by the structure identifying a place for isolation (room with bathroom), where they were assisted by health professionals equipped with PPE required by regional circulars. 
- In the event the swab was positive, without contraindications to the transfer, the woman was transferred to one of the identified reference centres (Hub). The sending hospitals contacted the reference Centres indicated above. Transports were carried out by the staff of the sending Centre.

- In the event the swab was positive and the transfer was contraindicated, a specific path was predisposed for the hospital management of confirmed cases, for obstetric assistance at delivery and for any situations in which there was a contraindication to the transfer of the pregnant woman.

- In the event the swab was negative, women were managed routinely, always wearing surgical masks.

- During breastfeeding, mothers had to wear surgical masks and gloves, till the confirmation of the laboratory negativity for nasopharyngeal swab.

- Birth partners, who were negative at the checklist, were allowed to stay in the delivery room only for labour and delivery and two hours after birth. They had to wear specific PPE (surgical mask, gloves, cap, overshoes, coat).

- Visitors (included birth partners) were not allowed to enter antenatal or postnatal wards (to ensure compliance with social distancing measures).

- All components of the staff were always equipped with PPE according to the risk of the procedure and the type of assistance.

- Live antenatal classes were stopped at the beginning of March and online sessions were offered.

- The offer of epidural was related to the availability of the anaesthesiologist often involved in concomitant requests for COVID emergencies

- The adoption of these labour and delivery management measures was shared with the Italian Superior Institute for Health, which at that time elaborated its guidelines ${ }^{5}$.

\section{Participants}

Participants were recruited through a consecutive mode, from the $25^{\text {th }}$ of March, 2020 to the $18^{\text {th }}$ of May, 2020, which corresponded to the new Ministerial decree for communicating less restrictive measures due to the drop of cases. Exclusion criteria were COVID symptoms or positive swab and the predefined criteria used in the pre-COVID cohort (language barrier, women who did not consent to the study, preterm $[<37$ weeks] or post-term [ $>42$ weeks] birth, previous CS, pre-labour caesarean section, pre-existing severe medical conditions or pregnancy complications [such as cardiac disease, haemoglobinopathies, renal disease, neurological disease, pre-eclampsia or complications), new-borns in poor condition at birth or who required any form of resuscitation.

At least 24 hours after delivery, women who met the inclusion criteria were invited to take part in the study by one of the six involved researchers, who explained to them the aim of the study, their involvement and asked them to sign the consent form. Women were enrolled every day of the week. Surveys were completed by mothers alone and returned before discharge.

\section{Ethical Consideration}

Ethical approval was obtained from the hospitals' Ethical Review Board (Approval number: 37/2018). Written informed consent was gained from all the participants.

5. Measurement tools

The BSS-R is a validated 10-items, self-report scale that was developed in the UK to evaluate women's satisfaction with birth starting from the original Birth Satisfaction Scale of 30 items. The Italian version of the BSS-R has been recently developed following an extensive translation process.

Data were collected through the Italian version of the Birth Satisfaction Scale Revised (I-BSS-R). 
Participants' perceptions were measured using a series of simple statements with a five-point Likert scale. Four of the items are reverse-coded (e.g., 'I found giving birth a distressing experience'). Three main themes that affect birth satisfaction are assessed throughout 3 Sub-Scales: quality of care provision - QC - (four items involving helping women to feel in charge of the labour, birth environment, support and relationships with health care professionals), women's personal attributes - WA - (two items concerning the ability to cope during labour, feeling in control, childbirth preparation and relationship with the baby) and stress experienced during labour - SE - (four items related to distress, obstetric injuries, receiving sufficient care, obstetric interventions, pain, long labour and baby's health). Socio- demographic, obstetric and intrapartum data (gestational age, onset of labour, pain relief used, oxytocin augmentation, length of active phase $>12 \mathrm{~h}$, method of fetal heart rate monitoring, mobilization, continuity of midwifery care, mode of birth and perineum outcome), were available from the birth register and the electronic records.

\section{Statistical analysis}

Continuous variables were described by mean and standard deviation and categorical variables by percentages. Descriptive statistics was performed on the whole sample, separately for each cohort and within each sub cohort on subsamples defined by classification variables. Comparisons between distributions of continuous and categorical distributions were obtained by hypothesis testing across cohorts and within each cohort. $\mathrm{T}$ test and Chi-square test were used for continuous and categorical variables respectively. p-values were two sided. Data management and statistical analysis was carried out by Stata software version 16.

\section{Results}

Among 158 women who gave birth during the study period, 27 (17\%) did not receive the questionnaire, one woman did not consent to participate, $15(9.4 \%)$ were not recruited for language barrier and $15(9.4 \%)$ underwent pre-labour CS. Thus, the study group (COVID) comprised 100 women (Fig.1) who were compared to a cohort of 277 women (pre-COVID) recruited in a previous study performed in 2018 in the same setting ${ }^{6}$, for a total of 377 women. Socio-demographic characteristics, obstetric history and intrapartum care variables are reported in Table 1 . No differences were reported in terms of maternal age, education, rate of employment, ethnicity and parity. A lower number of women attended childbirth classes (51\% pre-COVID versus $26 \%$ COVID, $\mathrm{p}<0.0001)$. Concerning intrapartum care, we found a higher rate of induction $[25 \%$ pre-COVID vs. $40 \%$, COVID p 0.004], fewer active phases $>12 \mathrm{~h}$ [15\% pre-COVID vs. $6 \%$ COVID, $\mathrm{p} 0.018]$, more acceleration with oxytocin [24.9\% pre-COVID vs. $35 \%$ COVID, p 0.05]. The rate of epidural analgesia was not significantly decreased ( $32 \%$ pre-COVID versus $27 \%$ COVID, p 0.30 ). Further, a reduced rate of one-to-one assistance, mobility during labour and rate of intermittent auscultation was registered.

The two groups were comparable also in terms of mode of delivery, showing the same rate of spontaneous delivery (89.5\% pre-COVID versus $90 \%$ COVID, p 0.69).

No differences were reported in terms of satisfaction at birth in the two groups (I-BSS-R mean 27.0, SD 5.3 in pre-COVID versus mean 27.6, SD 6.1 in COVID, p 0.348, Table 2), also for the three subthemes (Quality of Care Provision p 0.43, Women's personal attributes p 0.40, Stress experienced during labour p 0.13). Intrapartum variables that significantly reduced maternal satisfaction were the same in the two periods (Table 3 ): epidural analgesia ( $\mathrm{p}<0.0001$ vs. $\mathrm{p}<0.0001)$, prolonged active phases $>12$ hours $(\mathrm{p}<$ 0.0001 vs. $\mathrm{p} 0.0001)$, oxytocin administration $(\mathrm{p}<0.0001$ vs. $\mathrm{p}<0.0001)$ and operative delivery $(\mathrm{p} 0.0009$ vs. p 0.0019). On the other hand, induction impacted negatively on satisfaction (p 0.11 in pre-COVID vs. p 0.004 in COVID), while antenatal classes and episiotomy/intact perineum were no more correlated with satisfaction at birth in COVID era. The absolute lowest scores in COVID era were found in those women who underwent active phases $>12 \mathrm{~h}$ (mean score 19, SD 6 compared to the mean score in COVID 27.6) and CS in labour (mean score 17, SD 12 compared to mean score 27.6).

From a qualitative point of view, women were also asked to report which factor affected most their childbirth experience (Fig.2). 99 out of 100 women interviewed answered. 31\% complained about the fact that birth partners were allowed to stay only during labour and $2 \%$, specifically, complained about the absence of birth partners during induction. $26 \%$ reported that COVID pandemic did not affect their childbirth experience in 
any way. $16 \%$ was upset due to the general stressful situation of pandemic. Wearing the PPE during labour annoyed 13\%. $8 \%$ reported that, although upset, the support of the staff reassured them. $1 \%$ reported that the particular circumstance became an occasion for positive introspection. One was worried about the availability of epidural analgesia. Only one reported that she was worried about being infected by other patients.

\section{Discussion}

\section{Main Findings and Interpretation}

No difference in terms of mothers' satisfaction with their childbirth experience was demonstrated comparing those who delivered during COVID pandemic to those who gave birth before the pandemic, in the same context of a low risk maternity unit in Northern Italy. Coherently, a recent study pointed out that the psychological impact and anxiety of the COVID-19 epidemic seems to be more severe in women who are in the first trimester of pregnancy during the outbreak ${ }^{7}$. Concerning our practice, less women attended childbirth classes and this can be explained by COVID-related restrictions such as the conversion of live lessons into online modules to avoid social contacts. Taking into account this different modality, attending antenatal classes did not affect in any way satisfaction at birth in contrast to our previous investigation.

An increased rate of intervention was found (higher rate of induction, fewer active phases $>12 \mathrm{~h}$, more acceleration with oxytocin) and this can be due to the specific context of COVID pandemic. Desire to reduce antenatal controls, duration of labour, hospitalization and a reduced availability of epidural analgesia could have promoted a more interventional obstetric approach. Further, a slight reduction in midwives' assistance quality was observed (reduced rate of one-to-one assistance, lower mobility during labour and lower rate of intermittent auscultation). We can argue that this can be a consequence of the stressful environment during emergency, although it did not affect the global satisfaction of women.

Concerning intrapartum variables that could affect satisfaction, similarly to the perception before COVID ${ }^{6}$ and to other research ${ }^{8-10}$ we showed that lower birth satisfaction was experienced with interventions, such as epidural analgesia, active phases longer than 12 hours, oxytocin administration and operative delivery. However, the impact of induction of labour changed according to the period of interest. Specifically, it was not associated with satisfaction in women treated before the pandemic, whereas resulted associated with lower satisfaction in those treated during the emergency. This finding can be partly explained by the absence of birth partners before active labour and it is also confirmed by women's free reports on the experience in hospital during COVID ( $31 \%$ complained about the fact that birth partners were allowed to stay only during labour). This observation is also linked to another interesting finding, namely the absolute lowest scores in COVID era were in those women who underwent active phases longer than 12 hours and CS in labour, considering that both conditions are often related to prolonged times.

\section{Strengths and Limitations}

This is for our knowledge the first study on practice changing and satisfaction at birth during COVID pandemic. The possibility of comparing these women to a pre-COVID cohort from the same maternity unit and with the same methods is a point of strength in order to ensure homogeneity in obstetric care.

However, our study is not devoid of limitations. First, it was performed only in one hospital and, consequently, we can't know to what extent our findings are generalizable. Second, we continued to recruit women also in the first half of May 2020, when the global burden of the emergency started to decrease, thus probably determining a different perception. On the other side, the preventive measures adopted in the hospital remained unchanged for the whole duration of the study.

\section{Conclusions}

No differences were reported concerning global satisfaction at birth, although an increased rate of active intervention was noticed (higher rate of induction, fewer active phases longer than 12 hours, more acceleration with oxytocin). Induction, active phases longer than 12 hours and CS in labour were less tolerated in COVID 
era. This specific context can help us in analysing which factors can be improved in the assistance of women, in order to guarantee a good perception both in emergent and routinely scenarios.

\section{Disclosure of interests.}

There are no conflicts of interests to declare.

\section{Contribution to authorship}

$\mathrm{AL}$ had the original idea of the study. AI, AL and SF wrote the paper. LA performed the statistical analyses for this study. AN helped in the revision of the final version of the paper and reviewed the literature. AI, SM and DF collected data. All authors read and approved the final manuscript.

\section{Details of ethical approval}

Institutional review board approval waiving informed consent was obtained for this prospective study from the hospitals' Ethical Review Board (Approval number: 37/2018). Written informed consent was gained from all the participants.

\section{Funding}

No external funding was used in the conduct of this study.

\section{References}

1. Hinic K. Understanding and promoting Birth satisfaction in New Mothers. MCN. American J Matern Child Nurs, 2017;42:210-215.

2. Larkin P, Begley CM, Devane D. Women's experiences of labour and birth: an evolutionary concept analysis. Midwifery . 2009;25:e49-59.

3. Karlstrom A, Nystedt A, Hildingsson I. The meaning of a very positive birth experience: focus groups discussions with women. BMC Pregnancy Childbirth 2015;15:251.

4. Odone A, Delmonte D, Scognamiglio T, Signorelli C. Covid-19 deaths in Lombardy, Italy: data in context. Lancet Public Health2020;5:e310.

5. Narang K, Ibirogba ER, Elrefaei A, Abrao Trad AT, Theiler R, Nomura R, et al. SARS-CoV-2 in Pregnancy: A Comprehensive Summary of Current Guidelines. J. Clin. Med. 2020;9:1521.

6. Fumagalli S, Colciago E, Antolini L, Riva A, Nespoli A, Locatelli A. Variables related to maternal satisfaction with intrapartum care in Northern Italy. Women Birth 2020;S1871-5192.

7. Saccone G, Florio A, Venturella R, De Angelis MC, Locci M, Bifulco G, et al. Psychological impact of COVID-19 during pregnancy. Am J Obstet Gynecol 2020;223:293-295.

8. Fleming SE, Donovan-Batson C, Burduli E, Barbosa-Leiker C, Hollins Martin CJ, Martin CR. Birth Satisfaction Scale/Birth Satisfaction Scale-Revised (BSS/BSS-R): a large scale United States planned home birth and birth centre survey. Midwifery 2016;4: 9-15.

9. Rijnders M, Baston H, Schönbeck H, van der Pal K, Prins M, Green J et al. Perinatal factors related to negative or positive recall of birth experience in women 3 years postpartum in the Netherlands,Birth 2008;35:107- 116.

10. Çalik KY, Karabulutlu Ö , Yavuz C. First do no harm — interventions during labor and maternal satisfaction: a descriptive cross-sectional study. BMC Pregnancy Childbirth 2018;18:415.

\begin{tabular}{llll}
\hline Table 1 - Description of the study sample & Table $\mathbf{1}$ - Description of the study sample & & \\
\hline Variable & Variable & Overall $(\mathbf{n}=\mathbf{3 7 7})$ & $\mathbf{C}$ \\
Socio-demographic & & mean & 42.9 \\
& Maternal age (years) & $n$ & 4 \\
& & 160 & 4 \\
& Education (graduated) & 320 & 9
\end{tabular}




\begin{tabular}{|c|c|c|c|}
\hline \multirow{3}{*}{ Ostetric history } & & & \\
\hline & Parity (primiparous) & 211 & \\
\hline & Antenatal Classes (yes) & 168 & \\
\hline \multirow[t]{16}{*}{ Intrapartum Care } & & $\mathrm{n}$ & \\
\hline & Spontaneous Labour (yes) & 268 & \\
\hline & Active Phase $>12$ hours & 48 & \\
\hline & Midwifery Care & & \\
\hline & One to One & 371 & \\
\hline & Mobility & 361 & \\
\hline & Intermittent Ausculation FCF & 62 & \\
\hline & Intrapartum Interventions & & \\
\hline & Oxitocin (yes) & 104 & \\
\hline & Epidural analgesia (yes) & 117 & \\
\hline & Episiotomy (yes) & 92 & \\
\hline & Intact perineum (yes) & 45 & \\
\hline & Mode of birth & & \\
\hline & Spontaneous & 338 & \\
\hline & Vacuum assisted & 27 & \\
\hline & C section & 12 & \\
\hline
\end{tabular}

Table 2 - Satisfaction at birth

\begin{tabular}{|c|c|c|c|c|c|}
\hline & Item $(\mathbf{N})$ & $\begin{array}{l}\text { Overall }(n=377) \\
\text { mean }\end{array}$ & $\begin{array}{l}\text { Overall }(\mathrm{n}=377) \\
\text { SD }\end{array}$ & $\begin{array}{l}\text { Group } 1(n=277) \\
\text { mean }\end{array}$ & $\begin{array}{l}\text { Group } \\
\text { SD }\end{array}$ \\
\hline BSS-R Total & 10 & 27.1 & 5.6 & 27.0 & 5.3 \\
\hline Sub-themes & & & & & \\
\hline Quality of care provision & 4 & 14.1 & 1.9 & 14.1 & 1.8 \\
\hline Women's personal attributes & 2 & 4.7 & 2.0 & 4.6 & 2.0 \\
\hline Stress experienced during labour & 4 & 8.4 & 3.3 & 8.3 & 3.2 \\
\hline
\end{tabular}

\begin{tabular}{lll}
\hline Table 3 - Intrapartum variables I-BSS-R (Total) & Table 3 - Intrapartum variables I-BSS-R (Total) & Table \\
\hline & Group $\mathbf{~ ( n = 2 7 7 )}$ & Group \\
Variables & Mean & SD \\
Nulliparous & 26.28 & 5.78 \\
Multiparous & 27.77 & 4.68 \\
Antenatal classes (yes) & 26.32 & 5.59 \\
Antenatal classes (no) & 27.65 & 5.00 \\
Spontaneous labour & 27.26 & 5.13 \\
Induction of labour & 26.08 & 5.90 \\
Epidural (yes) & 24.7 & 5.95 \\
Epidural (no) & 28.06 & 4.66 \\
Active Phase $>12$ hours & 23.31 & 5.60 \\
Active Phase $<=12$ hours & 27.63 & 4.95 \\
Oxytocin (yes) & 24.20 & 6.04 \\
Oxytocin (no) & 27.89 & 4.76 \\
Spontaneous & 27.37 & 5.05 \\
Vacuum assisted & 23.21 & 5.92 \\
C section & 24.1 & 7.84
\end{tabular}




\begin{tabular}{lll}
\hline Table 3 - Intrapartum variables I-BSS-R (Total) & Table 3 - Intrapartum variables I-BSS-R (Total) & Table \\
\hline Episiotomy (yes) & 25.97 & 5.63 \\
Episiotomy (no) & 27.33 & 5.20 \\
Intact Perineum (yes) & 29.31 & 3.77 \\
Intact Perineum (no) & 26.66 & 5.45 \\
\hline
\end{tabular}

\section{Hosted file}

Figure 1.docx available at https://authorea.com/users/350998/articles/475673-childbirthexperience-and-practice-changing-during-covid-19-pandemic-a-prospective-cohort-study

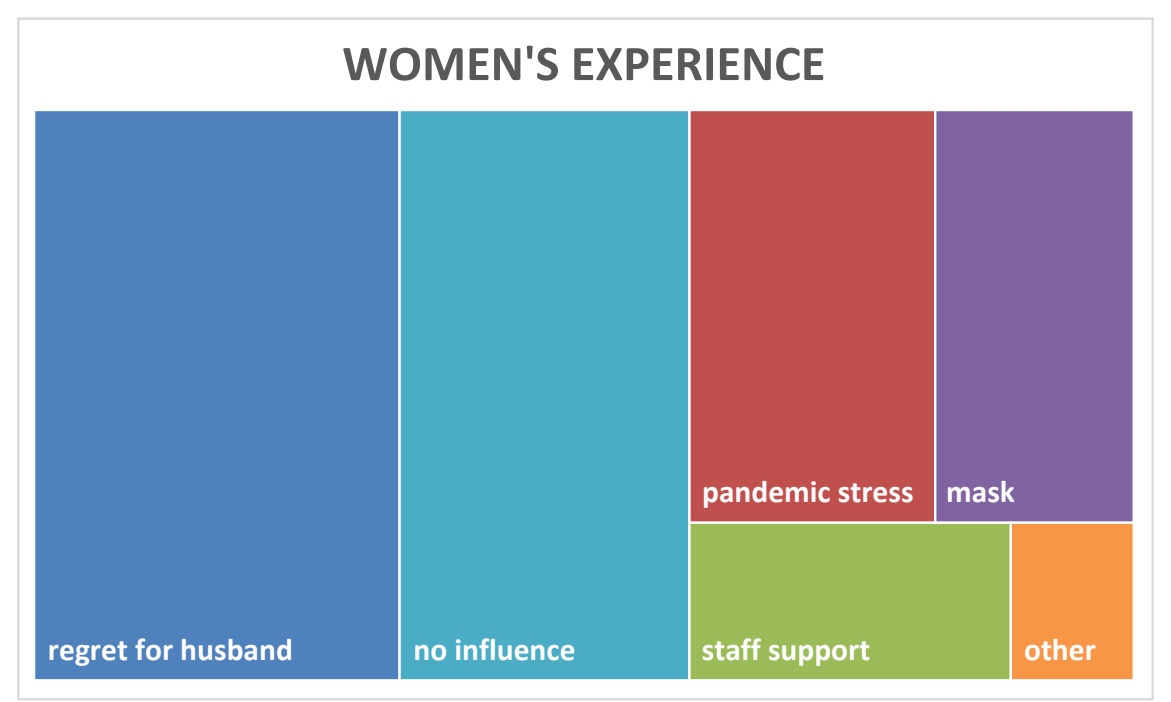

